\title{
Autonomous monitoring of nearshore geomorphology and hydrodynamics to assist decision making in coastal management, using shore-based radar systems: A case study on the Fylde peninsula, UK
}

\author{
Cai Oliver Bird, Marlan Maritime Technologies Ltd., Liverpool, UK \\ Alexander John Sinclair, Marlan Maritime Technologies Ltd., Liverpool, UK \\ Paul Simon Bell, National Oceanography Centre, Liverpool, UK \\ Marcus Phillips, Jacobs, UK \\ Carl Green, Wyre Council, UK
}

\begin{abstract}
Nearshore areas can be incredibly dynamic and therefore are difficult to monitor using traditional survey methods that typically provide only a snapshot of the physical conditions at the time of survey. These snapshot data are often re-used in the design of coastal defence interventions at a later date due to the high cost of data reacquisition over large areas. In this paper, a novel solution to large-area, continuous coastal monitoring is demonstrated. A shore-based radar station has been designed to collect image data within a $6 \mathrm{~km}$ range and derive a number of datasets including intertidal topography, subtidal bathymetry and surface currents. These data are then used in the development of beach management options to inform the Wyre Beach Management Outline Business Case which is currently being developed by Jacobs UK. At the time of writing, 10 weeks of data have been processed and have revealed the migration of sedimentary features along Rossall beach. Results derived using the radar system support anecdotal, previous scientific observations and modelled indications of sediment migration vectors, while revealing more detail on typical sediment fluxes along with system responses and recovery due to storm events. It is anticipated that this system will be the first of many permanent stations around the UK coastline, allowing a near-real time digital twin of the nearshore zone to be created and constantly updated. These datasets will form a crucial long-term evidence base for planning the most cost-effective coastal defence interventions and monitoring their effectiveness in response to variations in storminess, erosion and coastal squeeze being seen in the close vicinity of many vulnerable coastal areas.
\end{abstract}

\section{Introduction}

\section{Background}

Effective decision making for the management of flood and coastal erosion risk and in the design of coastal defence interventions requires a significant evidence base detailing coastal processes and the geomorphology around the region of interest (Burges et al. 2013). This requires monitoring of geomorphological and hydrodynamic (waves, currents and tides) regimes in dynamic nearshore areas over management timescales (decades), through frequent high resolution surveys and environmental monitoring.

Traditional survey technologies such as Differential Global Positioning System (DGPS), Terrestrial Laser Scanning (TLS), Multi-Beam Echo Sounders (MBES) and airborne Light Detection And Ranging 
(LiDAR) can deliver high resolution, high accuracy topography and bathymetry but are access-limited by weather, tide and logistical constraints. This, in addition to their high costs and length of mobilisation, make them unsuited to the task of continuous long-term monitoring. These limitations have resulted in the evidence bases used for decision-making often being comprised of temporally and/or spatially sparse physical datasets, such as annual LiDAR surveys in England \& Wales, supported by modelled hydrodynamics.

\section{Outline of this paper}

This paper describes the deployment of a shore-based radar system on the Fylde peninsula, northwest England to autonomously monitor intertidal topography, surface currents and subtidal bathymetry over a large area. Data processing performed to date includes the derivation of Digital Elevation Models (DEMs) of the intertidal zone over a period of 10 weeks and preliminary processing to create sample subtidal depth and surface current maps. This paper outlines data collection methodologies being used to build a long-term evidence base, with which to more effectively inform the beach management business case and assist ongoing coastal analytics following the construction of the $£ 63.2 \mathrm{~m}$ Rossall coastal defence scheme. This paper will also present preliminary analysis of the results in the form of morphological monitoring of Rossall Beach over a period of 10 weeks.

\section{Methodology}

\section{Deployment site - Rossall Seawall}

Work presented in this contribution builds upon that by Bird et al. (2018) where the first commercial deployment of these radar-based nearshore monitoring systems was described. Figure 1(a) illustrates the location of the system deployed on the Fylde peninsula and the instrumented range of the radar. A sample time exposure image created using 10 minutes of radar data from this site is also shown in Figure 1(b); range on this image is constrained to $4 \mathrm{~km}$ to show detail in the radar picture.
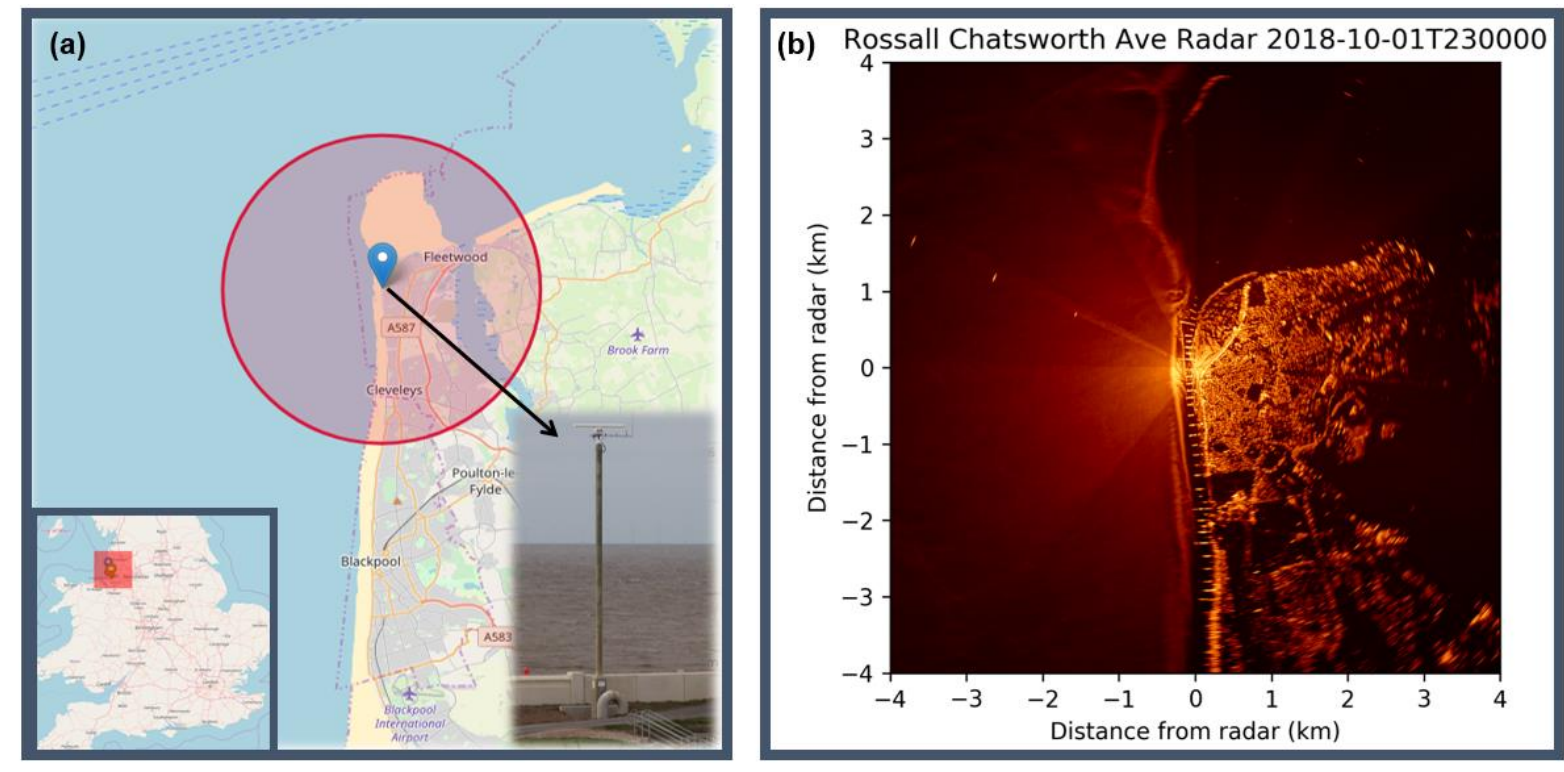

Figure 1 - (a) deployment site and radar instrumented range $(6 \mathrm{~km})$. (b) Sample time exposure image using 10 minutes of radar data collected on the day of installation. Image clearly shows the waterline and surrounding area.

The system deployed at Rossall Seawall makes use of existing infrastructure in the form of a $12 \mathrm{~m}$ CCTV column. A GEM Elettronica SuperNET2.2 m radar antenna is fixed onto a bespoke mounting plate allowing it to be bolted to the top of the lighting column. The system also mounts a meteorological sensor, a CCTV camera and an AIS receiver to enable validation of detected vessels during data analysis. 
The $9.41 \mathrm{GHz}$ antenna projects radio-frequency electromagnetic waves with a centimetre-scale wavelength. These waves interact with wind-generated capillary waves on the sea surface and produce strong backscatter signals through Bragg Scattering (Valenzuela et al., 1978), allowing waves and the breaking waterline to be clearly imaged with a marine radar.

\section{Raw data processing}

This radar system images the area within $6 \mathrm{~km}$ of the antenna every 3 seconds, and time-integrated images are created every 10 minutes. Sequences of these time-integrated images (Figure 1b), along with a record of tidal elevations are used to generate DEMs (Digital Elevation Models) of the intertidal area using the techniques detailed in Bell et al. (2016) and Bird et al. (2017). Radar images showing the progression of a moving wave field are fed to wave inversion algorithms which output subtidal bathymetry (Bell and Osler, 2011) and near-surface current vectors (Bell et al., 2012). Figure 2 illustrates the system data pipeline, from raw data collection and digitisation using the IRIS radar recording software, through the pre-processing of raw images that are fed to a selection of data processing methods (Figure 2(d)) and output as data products that are delivered to the end-user.

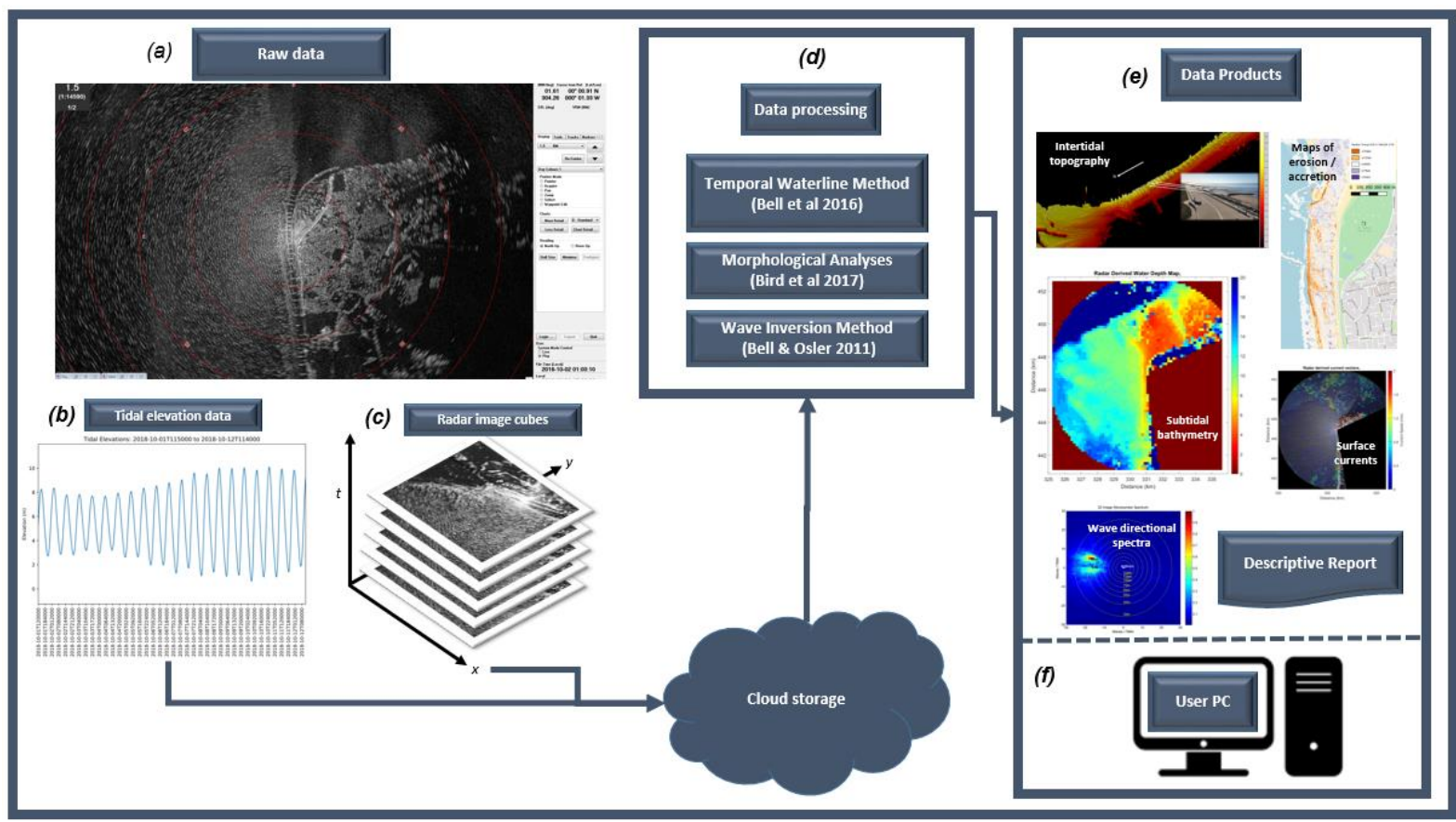

Figure 2 - flowchart of methods and outputs used in this paper. (a) Raw radar data are digitised and recorded using the IRIS radar software package. (b) Tidal elevation data recorded at a nearby tide gauge. (c) Radar data stacked into image cubes. (d) Methodologies used in this paper. (e) Data products produced by the methods used. (f) Data products are then sent to the user PC for further processing and analysis as required.

\section{Results}

\section{Radar-derived Digital Elevation Models}

At the time of writing, the radar monitoring system has been installed at Rossall for 10 weeks and raw image data have now been processed using the radar temporal waterline method (see Bell et al. 2016 for more detail on analysis methods and a discussion of the accuracy of the technique in respect to airborne LiDAR). Analysis to this point has resulted in a series of 5 intertidal elevation surveys, representing the average elevation relative to Admiralty Chart Datum at Liverpool over the course of 
each 2 week analysis period. Each survey derives a distribution of likely elevation values and uses a record of tidal elevations to select the most likely result. Radar image data are collected then stored in polar coordinates, which are then gridded onto a georeferenced $5 \mathrm{~m}$ Cartesian array.

The survey covers the intertidal zone along the Rossall beach between north Cleveleys and Rossall Point, and extends onto southern Morecambe Bay. Figure 3(a) shows an extract of the survey elevation map from a $3.5 \mathrm{~km}$ long stretch of beach adjacent to Rossall coastal protection scheme. To monitor erosion, this area is typically surveyed annually by the Environment Agency as part of the Regional Monitoring Programme using airborne LiDAR, while more frequent sparse beach profiles in the crossshore orientation are collected using DGPS systems, along with high resolution focussed TLS around groynes and at the toe of the seawall. Wyre Council carry out collection of these TLS data as part of the Cell 11 Regional Monitoring Programme (CERMs); the data already collected over a 20 year period by CERMs provides indicative longer term trends, but does not show critical short term storm induced reactions including scour at structures.

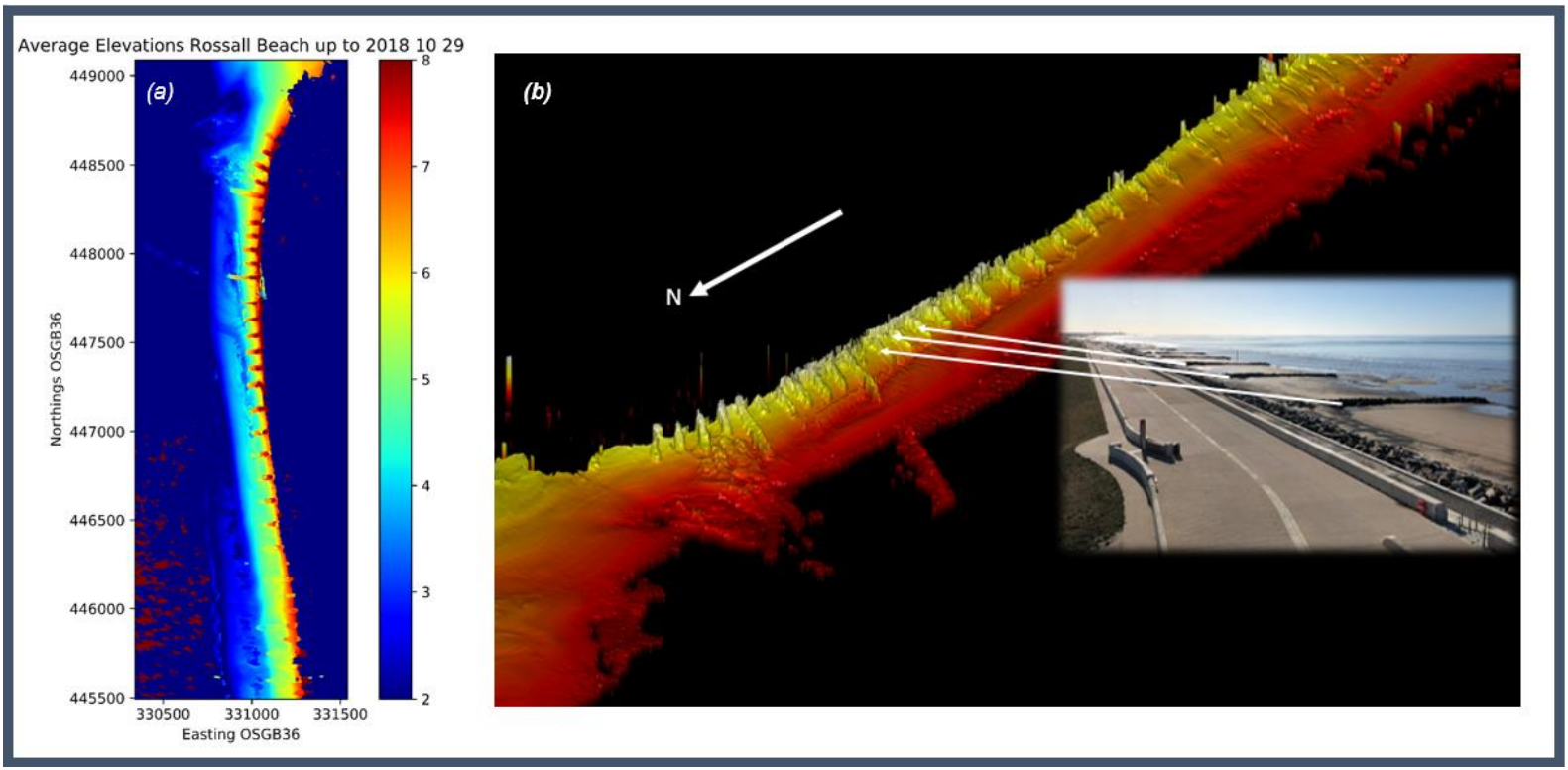

Figure 3 - (a) Radar-derived elevation map. (b) High resolution DEM showing location of groynes photographed.

The radar system supports these in-situ surveys by offering a wide area, high temporal resolution update on the condition of the intertidal zone and acting as a framework in which to contextualise the focused lower temporal resolution data. It is also intended to act as an early warning system to highlight any areas of erosion, scour, draw down or channel movements that require remedial intervention. Figure 3 (b) shows the same data set viewed from a 3D perspective, illustrating the groynes, the surrounding beach elevations and sediment volume that can be monitored.

\section{Monitoring geomorphological change}

Sequences of these intertidal DEMs are generated autonomously from continuously collected radar data. This allows morphological changes in the intertidal area to be monitored without deploying manpower to the coast or relying on data from national-scale monitoring campaigns whichmay not meet the needs of the local coastal managers. The objective of this analysis is to observe migrating sedimentary bedforms along the Rossall beach to ensure that sediment continues to move onshore and or northwards and is not adversely affected by the construction of the new coastal defences. Mathematical shoreline and coastal area modelling undertaken by Jacobs determined that the average annual net sand transport along this frontage was from south to north. A southwards or offshore movement of sediment was modelled to potentially occur during north-westerly storms and, if detected by the radar monitoring, will give cause for further investigation. These data must also be used to show 
that the beach elevations in the area immediately in front of the defences are remaining stable and that there are no significant areas of erosion. For example: scour around the groynes that may require immediate attention from the coastal authorities in the form of nourishment, or closer inspection using high resolution survey techniques.

The 5 surveys generated over consecutive 2 week periods are shown in Figure 4 along with a summary of key changes detected. A full spring tidal cycle (2 weeks) is used in the analysis, such that the greatest tidal range is imaged and elevations are captured at the upper and lower margins of the intertidal zone.

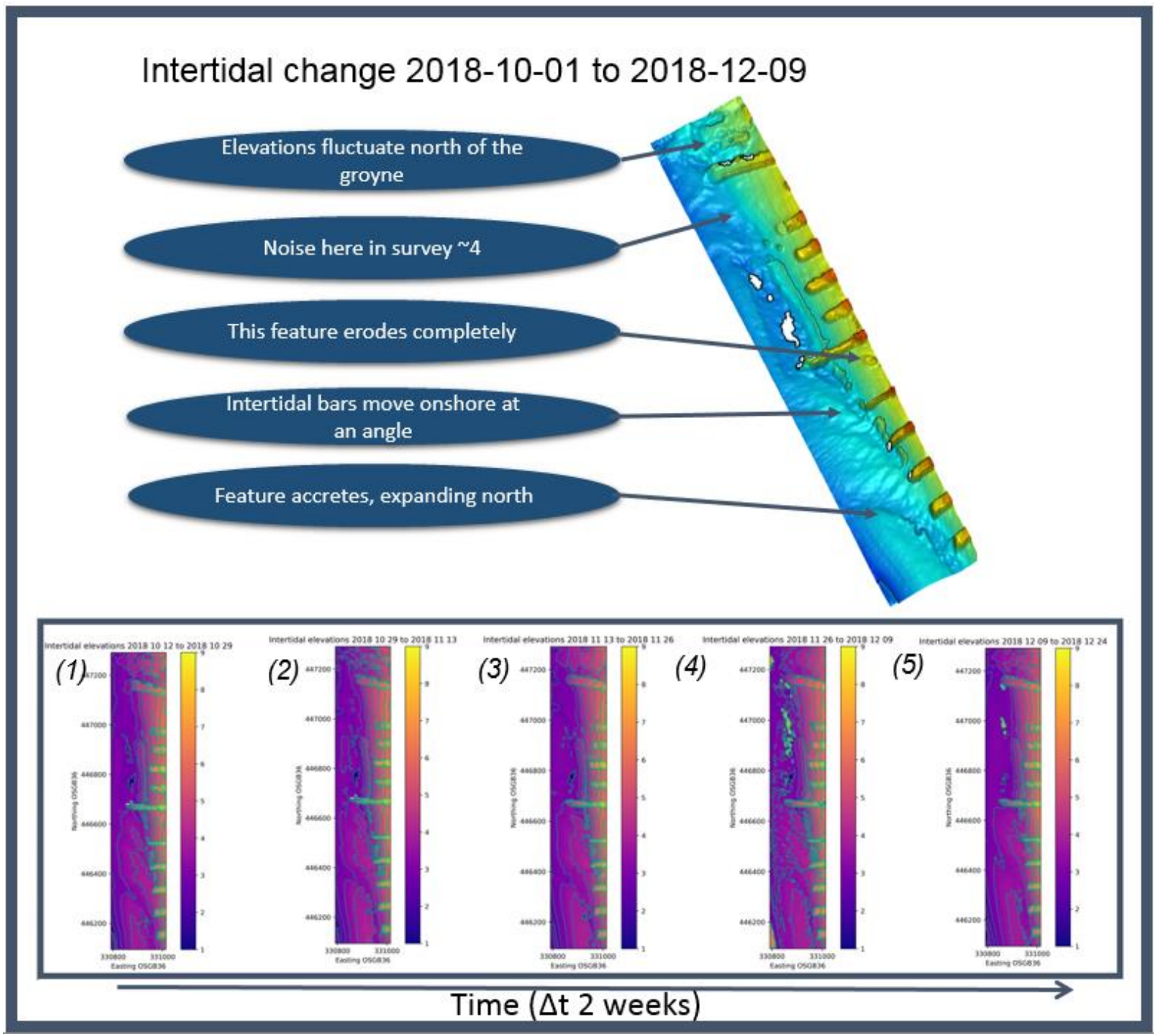

Figure 4 - DEMS from a subsection of Rossall beach extending $1000 \mathrm{~m}$ along-shore and $200 \mathrm{~m}$ cross-shore. Subplot shows consecutive surveys 2 weeks apart over a 10 week period. Key changes in the data are described in the callouts to the left.

There are processes taking place at a variety of spatial and temporal scales along this stretch of beach. The area to the north of the groyne indicated exhibits change at a much higher frequency than further south. Sedimentary changes occurring at high frequencies (e.g. change occurring over a single tide) are smoothed out, as such these plots represent gross changes in sediment volume.

While the results are stable from survey to survey over most of the area (evidenced by the groynes retaining their static elevations in each survey [more detail on the stability of results from survey to survey is given in Bell et al. 2016]), significant noise is present during the $4^{\text {th }}$ survey (November 2018). 
This is believed to be a result of calm weather during that period. Calm weather results in a lower signal to noise ratio between a wave breaking-induced waterline (of which the algorithm attempts to identify the temporal transition) and the dry sand of the beach immediately adjacent to the waterline. This results in poorer elevation estimates. This type of noise can be removed via a temporal smoothing process, weighted by the correlation coefficients that determine the confidence of the derived elevations (as described in Bell et al. 2016). However, in the interests of preserving data integrity and illustrating the limitations of this method in isolation, no such filtering was performed on these data.

A sequence of intertidal bars are clearly visible in Figure 4 and they migrate onshore rapidly during the 10 week analysis period. These bars also migrate at an angle relative to shore-parallel which gives the appearance of northwards migration. In addition to this system of bars, the larger sedimentary feature indicated accretes, expanding northwards by around $200 \mathrm{~m}$. This confirms local anecdotal knowledge, scientific observations and modelled predictions about this system with the dominant longshore current driving sediment northwards and migration of sediment onshore under typical conditions. See Miles et al. (2019) for a description of these intertidal bar systems along the Fylde coast that closely aligns with the radar-derived characteristics. Further analysis aims to show the effect of storms and changes in wave direction on sediment migration at this site.

\section{Detecting erosion; thresholds and alarms}

Some basic understanding of the system can be gleaned from the process of continuous monitoring, described previously. In order to capture gross changes in elevation resulting from erosion, accretion or interrupted sediment migration from the southern section of the beach, a simple set of thresholds can be implemented which ensure that the user is alerted to elevations that fall outside of a certain range.

Figure 5(a) illustrates this concept. With the radar-derived beach elevations shown as a semitransparent surface and an illustrative eroded surface below, these threshold surfaces can be used to define alarm zones that will alert a user when a given region is approaching the erosion threshold. Further allowing the user to understand particular problem areas and better manage beach nourishment operations. This region is highlighted in the photograph in Figure 5(c) and represents an area $300 \mathrm{~m}$ along-shore by $200 \mathrm{~m}$ cross-shore. The change in mean elevation over the 10 week analysis period is shown in Figure 5(b), along with erosion and accretion thresholds.

During the monitoring campaign, the difference in maximum mean elevation over the region of interest has so far been estimated as $0.081 \mathrm{~m}$; representing an estimated maximum volumetric change of 15,262 $\mathrm{m}^{3}$ over that area. To contextualise this, an area of nearby Cleveleys beach was nourished in 2014 with $10,000 \mathrm{~m}^{3}$ at a cost of $£ 115,000$. At Rossall, an allowance of $£ 2,587,500$ was made for $125,000 \mathrm{~m}^{3}$ over the next ten year period to reflect the modelled sediment losses at this scheme. It is an objective of this monitoring campaign to ensure that expensive beach nourishment is optimised, using a more significant evidence base. The locations and volumes of this sediment recharge can be informed by the erosion threshold alarms described above.

The migrating intertidal sedimentary features visualised and quantified by this system highlight that any given point on the beach is likely to experience a cyclic fluctuation in beach elevation associated with the migration of the bar-like features. Further, the precise arrangement of the bar-like features at the time of a wave event is likely to influence the vilnerability of the toe of the sea defences to wave attack. 


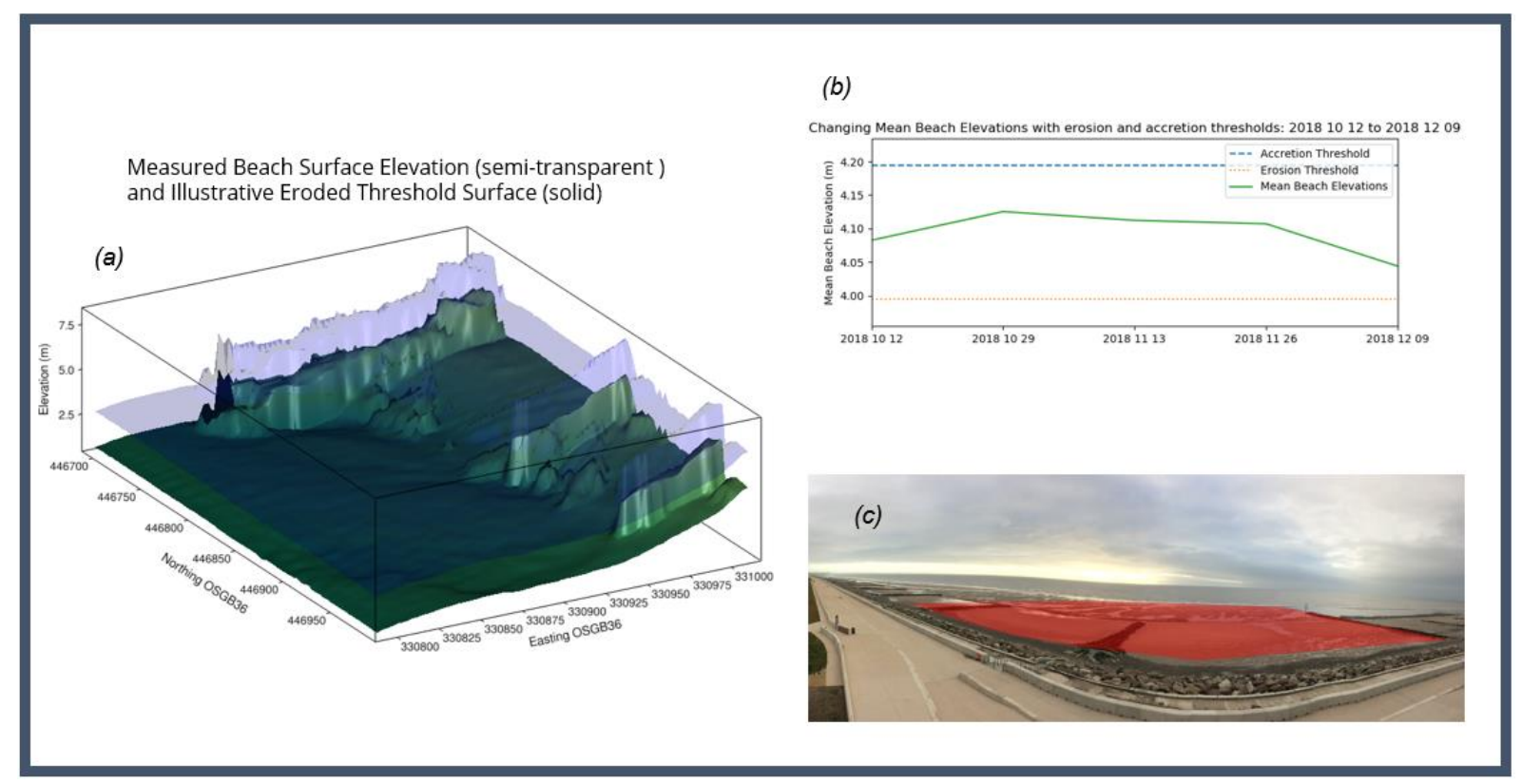

Figure 5 - (a) lllustrative eroded alarm threshold surface (solid) and beach surface overlaid (transparent). (b) Mean elevation change over 10 weeks (solid), with erosion (dotted) and accretion (dashed) thresholds. (c) Panoramic photograph of the area analysed in (a).

\section{Derivation of subtidal bathymetry and near-surface currents}

In addition to the detailed analysis of the intertidal elevations at this site, wave inversion techniques (Young et al 1985) have been applied to the raw data. Preliminary analysis of just under 6 hours of raw data, from high tide at 00:00hrs to low tide at 06:00hrs on $8^{\text {th }}$ December 2018 has resulted in a completed bathymetric map of the surrounding subtidal area out to $6 \mathrm{~km}$. Figure 6(a) shows the radar-derived water depth map relative to Admiralty Chart Datum (Lowest Astronomical Tide). The section of channel to the North, known as the Lune Deep is more than $40 \mathrm{~m}$ deep in places and shallows sharply onto Morecambe Bay.

Large subtidal sandbars are visible to the west; these will be monitored to determine if the source of sediment seen migrating onshore in Figure 4 originates from these banks. It is possible that they migrate into the channel or merge onto Morecambe bay, bypassing the beach at Rossall. A sewerage pipeline operated by a UK utilities company also runs under these sandbars into the Lune Deep and that route must also be monitored for erosion. Early warning of any potential erosion and uncovering of this asset will allow more cost-effective proactive mitigation and intervention planning.

Despite some shadowing of the intertidal area with respect to radar line of sight, the channel into Fleetwood from Morecambe Bay (visible at 333.5 east 450 north in Figure 6(a)) is able to be resolved using the wave inversion technique due to the waves being imaged at high tide; although the resolution may not be sufficient to fully resolve the depth of the narrow navigation channel at that range. The combination of the waterline and wave inversion techniques will eventually allow a merged Digital elevation model to be created representing the sub and intertidal depths seamlessly. 


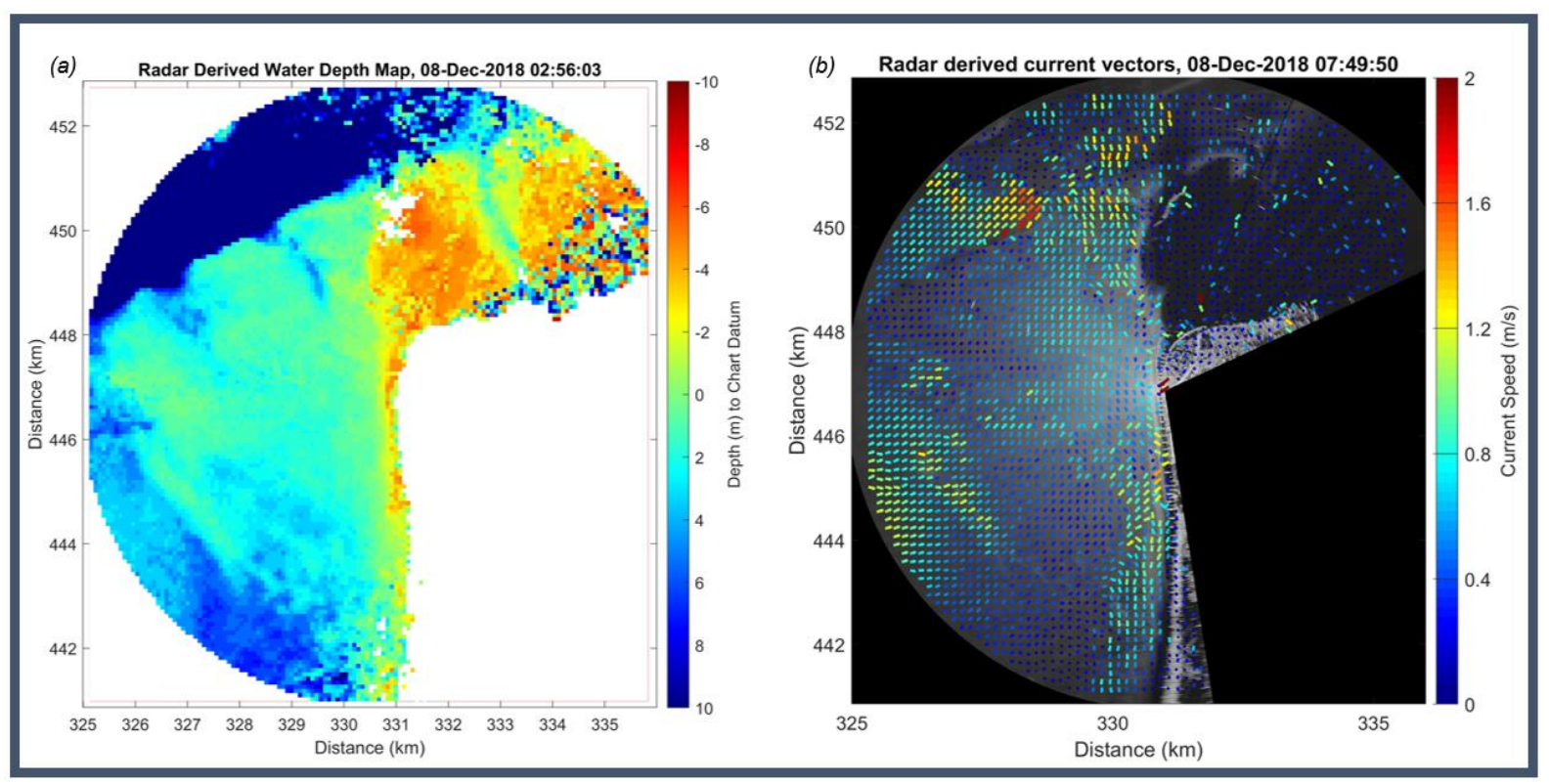

Figure 6 - (a) Radar-derived water depth map relative to Admiralty Chart Datum at Liverpool. (b) Radar-derived surface currents on the flood tide.

This technique derives depths at a much lower spatial resolution than the above described intertidal analyses and, while it is not therefore able to resolve local scour or small areas of erosion, the data act as a vital contextualisation to the high resolution intertidal data. Results from wave inversion methodologies are also able to resolve large changes in bathymetry and evolution of sand banks, so can provide timely data for hydrodynamic models and are therefore extremely useful. As reported in the local news (BBC 2016), a new gravel spit has also started to form at 3305 east 4490 north; monitoring the evolution of this spit will assist in public engagement with local coastal management issues.

In addition to an estimate of subtidal depth, a derivation of surface current direction and magnitude is also made using techniques described in (Hessner \& Bell, 2009). Currents are weak to the northeast in the sheltered zone and much stronger in the deep channel to the northwest and in the deeper water west of the subtidal banks. These strong onshore currents contribute to the sedimentary regime described earlier in this paper. The radar reveals a large spatial variation in currents across the analysis domain and will assist further modelling of interventions.

\section{Discussion}

\section{Results overview}

Results presented in this paper describe preliminary analysis carried out on an extensive dataset collected from a radar system on the Fylde Peninsula at Rossall. The analysis of intertidal elevations over a three month period have reinforced local expert knowledge and modelling by observing what appears to be steady onshore sediment migration; stable elevations across most of the surveyed area and sedimentary bedforms migrating northwards according to the dominant, typical longshore drift mechanisms.

Engineering interventions in the form of groynes and rock armour appear to be retaining sediment well. Further analysis of data captured during winter storms will assess the resilience of this system and measure the recovery rates of beach elevations following storms. Insufficient time has passed to make any inferences as to the seasonal stability of the system, however data presented in this paper have shown that large sediment movements occur on a sub-seasonal (two-weekly) timescale. These features have now been identified as coherent patterns through a sequence of radar surveys, and their movement and morphology can now be tracked. The coherence, rapid evolution and migration of the sedimentary features revealed by this technique are already bringing a new level of understanding of the dynamics of this particular beach. The authors anticipate that the ongoing nature and high update 
rate of the radar derived surveys will provide an unprecedented level of detail and understanding of the dynamics and response of this area to future natural forcings and managed interventions.

\section{System limitations}

The primary limitation of the system at Rossall is currently the limited absolute accuracy of the derived intertidal elevations with respect to ground truth. Elevations produced by this technique rely on good records of tidal elevation. However, due to the significant tidal gradient between the study site and the nearest tide gauge, the analysis described in this paper instead used elevations from the Port of Liverpool $50 \mathrm{~km}$ to the south. Therefore the absolute elevations described by these data will almost certainly be incorrect by some degree. However, the relative stability of the results between surveys is evidenced by the groynes in Figure 4 which do not vary greatly in elevation. Therefore changes seen in the radar-derived DEMs from survey to survey are good proxies for changing bed elevation at this site despite systematic errors in absolute elevations. Stability of the results is further evidenced by the high spatial coherence of the DEMs as each pixel elevation is derived independently from its neighbours and no spatial smoothing has been performed on the data presented. This issue is being addressed by the development of a tidal measurement solution that will be deployed at Rossall in 2019.

The issue of shadowing is prevalent in many areas of remote sensing such as airborne and ground based LiDAR, unmanned aerial vehicle (UAV) photogrammetry and synthetic aperture radar (SAR) satellite imaging. It is also a great issue in ground-based radar remote sensing. The tower at Rossall is mounted $12 \mathrm{~m}$ above ground level at the apex of the seawall cross section and thus it is possible to image between the groynes for several kilometres alongshore (as shown in Figure 3(b)). However, at ranges greater than $\sim 3 \mathrm{~km}$ the groynes begin to significantly shadow areas behind them, reducing the value of the radar-derived data at that range. This issue is compounded by the radar beam width footprint increasing with range, reducing the ability of a single radar system to resolve single target locations at range. This has the effect of 'smearing' a target over multiple pixels and therefore reduces the spatial accuracy of the radar system when attempting to image individual sedimentary features. These issues can be resolved by increasing the height of the antenna to alleviate shadowing; making use of multiple antennae to resolve targets better at range, and imaging shadow areas in a mutually complimentary manner.

\section{Further planned work}

Monitoring is to continue at this site, not only to better understand and characterise the morphology of migrating sedimentary features at the site, but also to continue to monitor the forcings of this migration through analysis of the hydrodynamic regime.

Absolute accuracies of the intertidal elevations are to be improved by instrumentation of the water surface elevations at this site. In turn, this will lead to a detailed comparison between local TLS and airborne LiDAR surveys with the radar-derived elevation data, to assess the potential for radar to replace the manual component of the survey campaign.

The real-time aspect of alarm thresholding as described in this paper will be further developed and tested with validation from targeted local photogrammetric and topographic surveys. The data pipeline and user interfaces that allow these early warning systems to be used must also be further developed and tested.

\section{Conclusion}

The radar system installed at Rossall on the Fylde peninsula is expected to be the first of a permanent, integrated regional network of these systems along the northwest coast of England. The system provides high spatial and temporal resolution observations of the physical processes and forces acting on the nearshore environment, including sediment transport pathways that are highly relevant to coastal management activities at this meso-scale. This information gives greater confidence to coastal managers to justify targeted interventions and, conversely, to defer intervention to be more costeffective. This would ideally then allow responsive management options and adaptation methods to be better explored and developed.

This monitoring campaign using radar-based remote sensing is an innovative beach management practice; continuous observation and data collection significantly de-risk beach management 
interventions. Interventions can be smaller and more targeted with the use of a high temporal resolution dataset that indicates regions in need of nourishment or closer observation. Costly beach nourishment interventions can be postponed while radar continuously monitors beach stability and recovery, representing significant cost savings on unnecessary or inefficient interventions. Adverse sediment migration such as the accretion of sediment on or around an important piece of infrastructure can be detected in advance of the event and allow mitigation measures to be put in place.

Over longer timescales, these data can feed into and be used to cross-validate ongoing modelling efforts at the site, improving the end model by providing greater information density than the previous temporally and spatially sparse datasets collected. In some cases, such as the radar-derived wide area surface current data, an equivalent dataset could not be collected by traditional techniques due to the logistical constraints and deployment costs of buoys and surface-mounted Acoustic Doppler Current Profilers (ADCPs).

The data collected from this site are also being analysed in collaboration with colleagues from the coastal team at Jacobs UK to support the development of beach management options to inform the Wyre Beach Management Outline Business Case, which is currently being developed. It is anticipated that a greater temporal and spatial density of data on coastal processes at the site will enable more efficient coastal management decisions and support ongoing interventions.

\section{References}

BBC News, 2016. https://www.bbc.co.uk/news/av/uk-england-lancashire-36249557/drone-footage-ofnew-island-near-fleetwood

Bell, P.S., Osler, J.C., 2011. Mapping bathymetry using X-band marine radar data recorded from a moving vessel. Ocean Dynamics, Vol61, pp. 2141-2156.

Bell, P.S., Bird, C.O., Plater, A.J., 2016. A temporal waterline approach to mapping intertidal areas using X-band marine radar. Coast. Eng. 107:84-101.

Bell, P.S., Lawrence, J. \& Norris, J. (2012) Determining currents from marine radar data in an extreme current environment at a tidal energy test site. In: Proceedings of the IEEE International Geoscience and Remote Sensing Symposium 2012. Piscataway, NJ, USA, IEEE, 7647-7650.

Bird, C.O., Bell, P.S., Plater, A.J., 2017. Application of marine radar to monitoring seasonal and eventbased changes in intertidal morphology. Geomorphology, Vol 285, pp. 1-15.

Bird, C.O., Bell, P.S., Sinclair, A.J., Truss, S.M., Plater, A.J., 2018. A Novel Technique for Nearshore Morphological Monitoring Using Marine Radar: Initial Deployment and Preliminary Survey Results. Coasts, Marine Structures and Breakwaters 2017, pp.263-266.

Burgess, K., Pontee, N., Wilson, T., Lee, S.C., Cox, R., 2013. Steart Coastal Management Project: engineering challenges in a hyper-tidal environment. Coasts, Marine Structures and Breakwaters 2013, Aberdeen, 17 - 20 September 2013.

Hessner, K., \& Bell, P. S. 2009. High resolution current \& bathymetry determined by nautical X-Band radar in shallow waters. In OCEANS 2009-EUROPE. 1-5. IEEE.

Miles, A., llic, S., Whyatt, D., James, M.R., 2019. Characterising beach intertidal bar systems using multi annual LiDAR data. Earth Surface Processes and Landforms. doi: 10.1002/esp.4594.

Valenzuela, G. R. 1978. Theories for the interaction of electromagnetic and oceanic waves-A review. Boundary-Layer Meteorology, 13(1-4), 61-85. 